\title{
The Signal Detection Model of AM-EAS Based on Optimized SVM with Improved AFSA
}

\author{
Yanan Geng \\ School of Information Engineering, Zhengzhou University, Zhengzhou 450001, China \\ 1922709149@qq.com
}

Keywords: Artificial fish swarm algorithm; SVM; AM label; Detection rate; Real-time

\begin{abstract}
In order to improve the detection rate of AM-EAS system, The paper puts forward an new signal detection model (IAFSA-SVM) combined with the Improved Artificial Fish Swarm Algorithm (IAFSA) and Support Vector Machine (SVM) .the paper analyzed the advantage and defect of AFSA, and proposed the improved scheme, The simulation results show that IAFSA than AFSA, GA and PSO has a better ability of parameters optimization about SVM, The model based on the optimized SVM compared with the traditional AM label detection algorithm has markedly improved in detection rate and false positives, and satisfies the requirement of real-time detection in engineering application.
\end{abstract}

\section{Introduction}

The Electronic Article Surveillance [1] (AM-EAS) is designed by the Principle of Tuning Fork; it's widely applied in the retail industry. About the study of AM-EAS, The focus of the research is electromagnetic radiation and the detecting algorithm of label. The current domestic products are mostly using the label detection algorithm with FFT and SNR. Although the two algorithms show the characteristics of high sensitivity and instantaneity, but the detection rate is low. The researchers tried to merge the two algorithms and adopted the detecting method of double threshold, but the omission phenomenon is serious in practical application.

In order to improve the detection rate of AM-EAS, the paper puts forward a model (IAFSA-SVM) of tag signal detection that is a blend of the Improved Artificial Fish Swarm Algorithm [2] (IAFSA) and the Support Vector Machine [3] (SVM). The simulation based on UCI data shows that the IAFSA compared with the ASFA, Genetic Algorithm(GA) and Particle Swarm Optimization [4] (PSO) reflects a better ability of parameters optimization, and the optimized SVM model's accuracy is higher. IAFSA-SVM model compared with FFT and SNR reflects the characteristics of the higher detection rate and farther detection distance in practical test, the module also can meet the requirements of real-time detection.

\section{Material and Methods}

The Support Vector Machine. The samples could be divided into two categories by hyperplane that keeps the largest interval of sample's classification [5].There are linearly separable data:

$\left(x_{i}, y_{i}\right), x_{i} \in R^{n}, y_{i} \in\{1,-1\}, i \in 1, \ldots \ldots n$

The Classification function was:

$$
f(x)=w^{T} x+b
$$

Due to the margin was the largest, so the classification function could be converted into the problem of quadratic programming.

$$
\left.\begin{array}{c}
\min \frac{1}{2}\|w\|^{2}+C \sum_{i=1}^{1} \xi_{i} \\
y_{i}\left(w^{T} x_{i}+b\right) \geq 1-\xi_{i} \\
i=1, \ldots, n, \xi_{i} \geq 0
\end{array}\right\}
$$


The $\mathrm{C}$ and the $\xi \mathrm{i}$ could adjust the error, the function could be expressed as a form of Lagrange function.

$$
L\left(w, b, \xi_{i}\right)=\frac{1}{2}\|w\|^{2}+C \sum_{i=1}^{1} \xi_{i}-\sum_{i=1}^{1} \alpha_{i}\left[y_{i}\left(w^{T} x_{i}+b\right)-1+\xi_{i}\right]-\sum_{i=1}^{1} \gamma_{i} \xi_{i}
$$

According to nonlinear and categorical data, the kernel function could map the data to the feature space of higher latitudes, and then, the data could be linear division by function in the higher space. Under the condition of smoothness assumptions and insufficient data, the Radial Basis Function (RBF) had a better ability of learning and generalization [6], so the paper choses the RBF.

$$
K\left(x_{1}, x_{2}\right)=\exp \left(-\frac{\left\|x_{1}-x_{2}\right\|^{2}}{2 \sigma^{2}}\right)
$$

Through the Lagrange Multiplier method, the final classification function was:

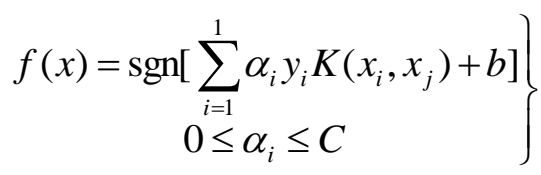

After the kernel function was determined, the classification function had two uncertain parameters, the best combination of $\mathrm{C}$ and $\sigma$ was the key to the function's performance.

The Artificial Fish Swarm Algorithm. The Artificial Fish Swarm Algorithm (AFSA) was a new type of swarm intelligence algorithm, it could simulate the fish's behaviour of Foraging, Following, Cluster and Random. The Fish would gather to rich nutrients in the water by individual competition and coordination, and achieve global optimization.

The function was represented as:

$$
\begin{aligned}
& V_{\text {next }}=x_{i}+\text { Visual } \cdot \theta, i=1,2, \ldots n \\
& X_{\text {next }}=\frac{X_{i}-X}{\left\|X_{i}-X\right\|} \cdot \text { Step } \cdot \theta+X
\end{aligned}
$$

The $\theta$ was a random number $[-1,1]$, the initialized parameters of algorithm included: the fish $\operatorname{scale}(N)$, the distance of view (Visual), the distance of step(Step), the crowding factor $(\delta)$, the number of iterations(Number), etc.

The Improved Artificial Fish Swarm Algorithm. It was found that: the fixed Step and Visual would affect the convergent speed and accuracy of algorithm.so the paper has made the improvement.

1. Setting two different parameters of the Visual. The IASFA will set the Cluster and Following to be a relatively bigger value of Visuall, and the Foraging to be a smaller value of Visual2. two different Visual can improve the convergent speed and precision.

2. Introducing fluctuant factor: $R$. the $R$ can make the Step and the Visual to keep dynamic changes in the iterative process, Step $=R *$ Step, Visual $=R *$ Visual.

3. Introducing the mechanism of elimination. When the number of iteration is more than half, if the objective value of the someone is lower than the default threshold, then the fish will be eliminated, and release the memory space. The mechanism of elimination can effectively improve the convergent speed and reduce the complexity.

The model that integrates IASFA and SVM is as follows Fig. 1.

The Signal Feature. The commodities are attached to the electronic label, the resonance frequency of the tag and the carrier frequency is consistent, the label will produce resonance phenomenon when it passes the detection area, if the detector detects resonance signal, that means some goods have been out of the mall without purchase ${ }^{[7]}$. This article is based on the AM-EAS system with $58 \mathrm{KHz}$ to study. Due to reasons such as signal's coupling and reflection, the detector will receive the continuous noise signal, the actual detecting signal is as shown in Fig. 2. 


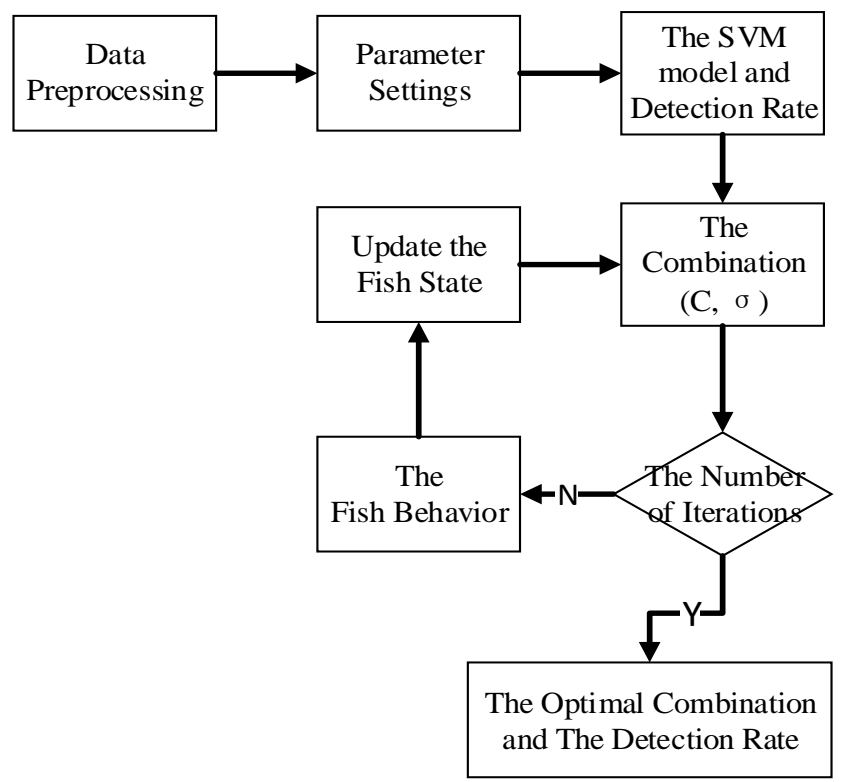

Figure 1. The IAFSA-SVM mode

Through analysing the characteristic of the signal frequency, it can be found that the noise's power spectrum concentrated in $60 \mathrm{KHz}$, the maximum modulus is 36.68 .

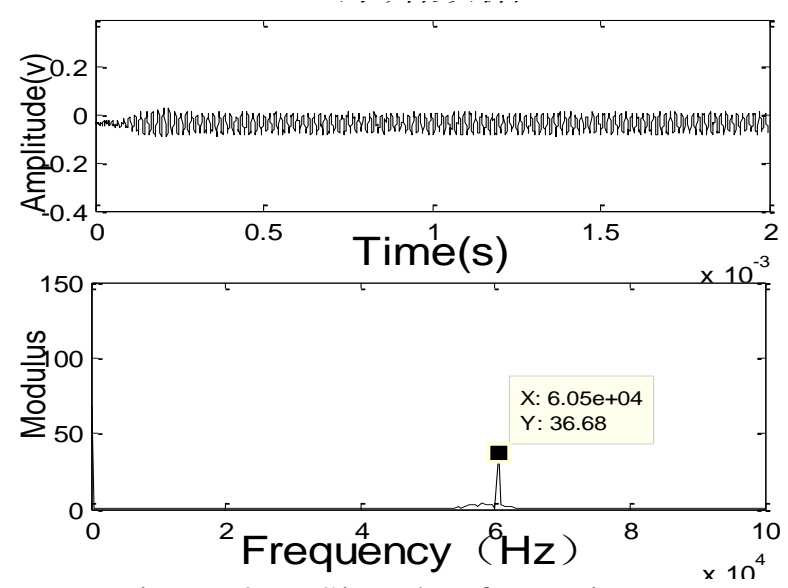

Figure 2. Signals of Inactive Tag

The AM-EAS system's testing width is between $1.0 \mathrm{~m}$ and $1.5 \mathrm{~m}$, the distance between tags and antenna is the closer, the amplitude of resonance signal is the greater, when the distance of tags and the receiving antenna is $0.8 \mathrm{~m}$, the actual detecting signal is as shown in Fig. 3.

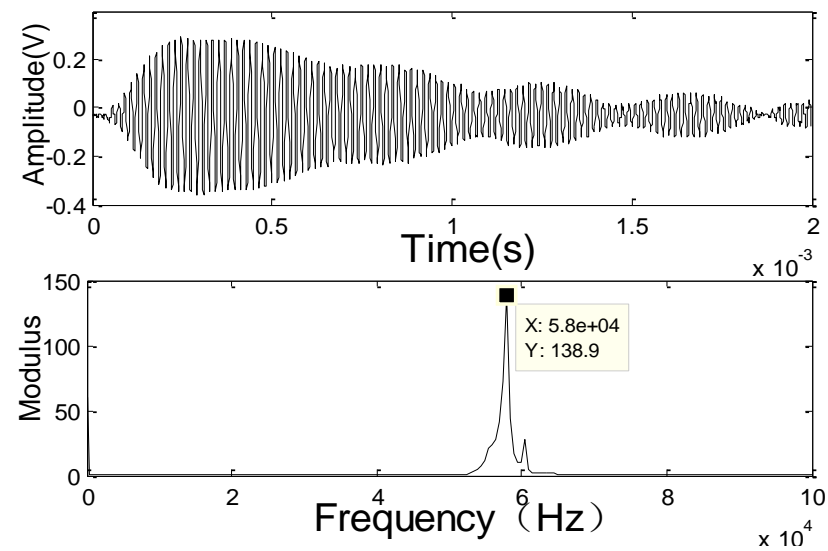

Figure 3. Signals of Activate Tag 
According to the characteristic of the signal frequency, we can be found that the noise's power spectrum concentrated in $58 \mathrm{KHz}$, the maximum modulus is 138.9. Considering the reliability of the signal and the complexity of algorithm, when the distance between label and antenna is at $0.5 \mathrm{~m}, 1 \mathrm{~m}$ and $1.5 \mathrm{~m}$, the experiment can gather the signal and extract intermediate frequency which maximum modulus and SNR as a feature vector, so the SVM's input vector contains nine parameters, The SVM model would divide the data into two categories including +1 (Alarming) and -1 (No Alarming).

\section{Results}

The Comparison of Optimization Algorithm. The simulation data is Iris, Yeast, Optical, IS, Seeds and Segment from the UCI Database [8-9], the sample properties are shown in Table 1. respectively, using PSO, GA, AFSA and IAFSA to find the optimized parameters of SVM, the result of each group is the average in experiment many times, the simulation results are shown in Table2.

Table 1 The Property of Data

\begin{tabular}{cccc}
\hline Sample & Length & Dimension & Classification \\
\hline Iris & 200 & 4 & 3 \\
Yeast & 730 & 8 & 10 \\
Optical & 3600 & 64 & 10 \\
Segment & 850 & 19 & 7 \\
IS & 420 & 19 & 7 \\
Seeds & 300 & 7 & 3 \\
\hline
\end{tabular}

The initialized parameter in IASFA [10]: the number of iterations was 50, the number of colony was 10 , the number of temptation was 30, the number of Crowding was 0.625 , the number of Visuall was 0.8 , the number of Visual2 was 0.4, the number of Step was 0.1, the number of Volatility was 0.05 , the number of Elimination was 0.6 , the Value range of $C$ and $\sigma$ was $[0.01,10]$.

From the simulation results, we can be found that the IAFS A has a stronger ability of parameter optimization than GA, PSO and AFSA, and the homologous model's learning ability is better in the situation of the big data or the multi-classification,

Table 2 The Comparison of Algorithm's Performance

\begin{tabular}{|c|c|c|c|c|c|c|c|c|}
\hline & PSO & GA & AFSA & IAFSA & PSO & GA & AFSA & IAFSA \\
\hline Iris & \begin{tabular}{|l|}
98.4 \\
\end{tabular} & 98.32 & 98.67 & 99.12 & $(6.6504,0.4591)$ & $(5.071$ & $(5.2$ & $(5.4$ \\
\hline Yeast & 77.42 & 75.98 & 78.85 & 80.47 & $(7.0871,0.9837)$ & $(6.8943,0.4424)$ & $(6.7864,0.842)$ & $(6.992,1.024)$ \\
\hline Optical & 98.87 & 99.65 & 99.65 & 99.68 & $(1.321,0.2385)$ & $(0.6030,0.4158)$ & $(2.6523,0.4931)$ & $(1.946,0.529)$ \\
\hline Segment & 84.84 & 84.23 & 87.64 & 87.72 & $(6.979,0.3503)$ & $(5.505,0.1509)$ & $(9.296,0.6097)$ & $(7.243,0.384)$ \\
\hline IS & 92.21 & 91.42 & 92.91 & 93.83 & $(6.758,0.1382)$ & $(5.1707,0.2294)$ & $(3.4245,0.1623)$ & $(5.296,0.201)$ \\
\hline Seeds & 97.3 & 95.53 & 98.12 & 99.26 & $(9.3367,0.8583)$ & $(5.9704,0.2905)$ & $(7.982,1.000)$ & $(7.845,0.912)$ \\
\hline
\end{tabular}

The Comparison of Detection Model. The experiment's data is a total of 2072 groups in AM-EAS testing; uniform random sampling 1072 groups from the total are as the training sample, the remaining 1000 groups are as test samples. The IAFSA has calculated the optimized combination of the SVM's parameters about AM-EAS was $(5.358,0.2285)$, respectively, using FFT, SNR and the trained SVM model. The simulation results were as follows Table 3. 
Table 3 The Comparison of AM-Label Detection

\begin{tabular}{cccccc}
\hline Algorithms & $\begin{array}{c}\text { Elapsed time } \\
\mathrm{ms}\end{array}$ & $\begin{array}{c}\text { Distance } \\
\mathrm{m}\end{array}$ & Misinformation & Omission & Detection Rate \\
\hline FFT & 2.3 & 1.36 & 2 & 0 & 96.49 \\
SNR & 1.2 & 1.15 & 5 & 1 & 93.82 \\
IAFSA-SVM & 4.6 & 1.42 & 0 & 0 & 99.75 \\
\hline
\end{tabular}

The experimental results show that the IAFSA-SVM model has a better detection rate and distance than FFT and SNR. The average of elapsed time is about $4.6 \mathrm{~ms}$, the SVM model also can satisfy the requirement of real-time monitoring in the existing DSP or FPGA.

\section{Conclusion}

In view of the problem of low detection rate and serious omission in the AM-EAS, the paper puts forward a detective model that is the combination of IASFA and SVM. The simulation results show that the IASFA compared with the ASFA, GA and PSO had a better capability of searching in determining the optimal parameters of the SVM; the IAFSA-SVM had improved significantly than traditional label detective algorithm in detecting distance and rate, etc. at the same time, it could meet the requirements of real-time system. The study of IAFSA-SVM in AM-EAS is not only enriching the application of the SVM, but also provides a new solution for other signal recognition.

\section{References}

[1] Khan L, Awad M, Thuraisingham B: The VLDB Journal, Vol.16 (2007) No.507.

[2] Suykens J A K, Vandewalle J: Neural Processing Letters, Vol.9 (1999) No.293.

[3] VapnikV: The Nature of Statistical Learning Theory (New York Publications, Wiley, 1998).

[4] Jayadcva R, Khemchandani S C: IEEE Trans on Pattern Analysis and Machine Intelligence, Vol.29 (2007), No. 905.

[5] Burges C J C.A Tutoria 1 on Support Vector Machines for Pattern Recognition [J].Knowledge Discovery and Data Mining,Vol.2(1998) No.121.

[6] Guo Guo-dong, Stan Z Li, Kap Luk Chan: Image and Computing, Vol.19 (2001) No.631.

[7] Zhang Xuegong: The Nature of Statistical Learning Theory (Tsinghua University Publications, Beijing, 2000).

[8] M Aminian, F Aminian: IEEE Transactions on Instrumentation and Measurement Vol.56 (2007) No.1546.

[9] Gussous I, Cornuz J, Paccaud F: Swiss Medical Weekly, Vol.137 (2007) No.304.

[10]Beadsmoore C. J, Screaton N. J: European Journal of Radiology, Vol. 45(2003) No.8. 